\title{
Towards Knowledge Driven Decision Support for Personalized Home-based Self-management of Chronic Disease
}

\author{
Jun Qi \\ School of computer science and informatics \\ De Montfort University \\ Leicester, UK \\ p14187732@myemail.dmu.ac.uk \\ Wolfgang Leister \\ Norwegian Computer Centre \\ Oslo, Norway \\ wolfgang.leister@nr.no
}

\begin{abstract}
The use of ICT technologies to facilitate self-management for patients with chronic diseases attracts increasing attention in smart healthcare. Existing research has mainly focused on sensing and data processing technologies with little work on decision support mechanisms and systems. In this paper, we propose a home-based decision support system based on a wide range of assessment metrics from medical assessment, social and psychological evaluation to behaviour analysis to help self-manage rehabilitation and wellbeing in a personalized manner for different patients. This paper develops semantic models for describing patients, their conditions, and inference mechanisms for decision recommendations. The research is undertaken in the context of mobile user self-management for Spondyloarthritis (SpA) patient, a case study is used to demonstrate the principles.
\end{abstract}

Keywords - ontology; decision support; SWRL inference; patient self-management; Spondyloarthritis

\section{INTRODUCTION}

Chronic disease is the main cause of mortality in the last few decades. In developed countries, all parts of population are affected by chronic conditions [1]. Chronic disease is a common health problem and many of them are not curable, which usually leads to costly rehabilitation and reduced quality of life. Some chronic diseases are preventable and can be effectively controlled. Chronic disease management systems including pharmaceutical and non-pharmaceutical interventions are designed to maintain patients' health conditions and reduce the risk of morbidity. Patients with chronic disease normally need longterm and intensive interactions with various healthcare professionals to closely monitor, assess and thus control conditions. At the moment, this is mainly conducted manually by clinicians at health centres, requiring patients to visit hospitals regularly. However, this practice has many problems, e.g., medical testing is time-consuming; assessment is subjective; frequent visits to hospital are costly and not sustainable; and less frequent visits may miss opportunities for improvement so that impact wellbeing of the patients [2].

\author{
Liming Chen \\ School of computer science and informatics \\ De Montfort University \\ Leicester, UK \\ liming.chen@dmu.ac.uk \\ Shengxiang Yang \\ School of computer science and informatics \\ De Montfort University \\ Leicester, UK \\ syang@dmu.ac.uk
}

Personalised self-management systems have the goal to improve the quality of live (QoL) of the patients. They need to deal with a variety of patient conditions using smart monitoring, sensor technologies, objective and subjective assessment, treatment plans, and guidelines. The information given by the self-management system needs to be tailored to patients, carers and relatives of the patients, and health personnel. A self-management system can only take decisions that are described within the treatment plan. Diagnosis, clinical decisions done by the health personnel, and changes in medication are not part of a self-management system.

This work describes a personalized chronic patient self-management system (CPSMS) for Spondyloarthritis (SpA), a family of inflammatory rheumatic diseases that cause arthritis [3]. The proposed CPSMS will be used in the residential environment of the patients, but also be used for improving communication between patient and health personnel. The CPSMS is able to support the patient's self-management via knowledge description and inference for both patients and their conditions by providing a set of nonpharmacological treatment plans or suggestions. This will help patients to keep better control on these disease, and, at the same time, reduce the number of hospital visits.

The remainder of this paper is organized as follows: Section II introduces related work and background of clinical personalized decision support. Section III presents the system architecture and its main functions and their interplay. Section IV describes in detailed ontological modelling and decision support mechanism. Section V illustrates the usage of the system in a use scenario, followed by a conclusion in Section VI.

\section{RELATED WORK}

An ontology is a logic-based organizational structure for knowledge [4], defines concepts, properties, and relationships and supports instancebased reasoning. The $\mathrm{W} 3 \mathrm{C}$ Web Ontology Language (OWL) is a semantic web language for authoring ontologies, including sublanguages OWL Lite, OWL DL, and OWL Full [5]. OWL provides 
users with an expressive formalism for knowledge modelling and representation which supports computational completeness, decidability and practical reasoning algorithms.

Ontology-based decision support systems have been studied and deployed in various scenarios over the past decade. Abidi et al. [6] developed clinical practice guideline and decision support system to support family care for breast cancer patients in terms of semantic modelling and logic based inference. Riaño et al. [7] used an ontology based approach to develop two personalized procedures for chronical patient healthcare, including anomalous detection, missing data and preventive actions. Another example is shown by Paganelli and Giuli [8] with which the context semantic modelling and reasoning were provided as monitoring system for chronic patients. Thomas et al. [9] made use of asthma treatment guidelines to provide the physician with disease assessment and recommendations on the basis of objective functional patient testing and case based treatment. Martínez-García, et al. [10] discussed a knowledgebased system to support healthcare personnel to help patients manage depression. In addition to the research methods mentioned above, some ontology based open resources of decision support system that can share and reuse domain knowledge are already available [11]. Some ontological models have been widely used in the medical terminologies, such as ONIONS [12-14], Open Biological and Biomedical Ontologies Foundry [15], OGMS [16], Open biological and Biomedical Ontologies (OBO) [17] etc.

Most literature in ontology-based decision support systems have focused on remote monitoring, alerting patients to visit physicians when their conditions are under severe situations. Few of them are able to automatically provide nonpharmacological plans and recommendations for symptom management on a daily basis. In our work, we are attempting to resolve this problem through ontological construction and SWRL rule inferences, producing a series of recommendations to both patients, carers, and health professionals presenting treatment.

\section{THE CPSMS ARCHITECTURE}

Three main layers can be categorized in our system, which are the assessment layer, the model layer and the application layer, as shown in Figure 1. Heterogeneous information scattered in the assessment layer are from expert knowledge, sensor-based data and patient's self-report assessment in the assessment layer. The expert knowledge can be extracted from clinical guidance, i.e., health care literature, existing archetype definitions [18], or from health personnel's knowledge. To bring together information from disparate sources, ontologies are used to map a real world entity to an abstract model in digital world, i.e., conceptualizing and specifying entities in the assessment layer as ontological expression of concepts or classes in the model layer. A number of concepts or classes, instances, relations have been developed to characterize the $\mathrm{SpA}$ domain knowledge.

To support personalized decision support, a set of rules in inference engine extracted from the domain expert knowledge should be defined for each patient in the application layer. The outcomes of the inference engine are used to support decision making with the treat-to-target principle [19], an individual treatment procedure for patient selfmanagement. The result of the self-assessment will be compared with the target either by health personnel or the underlying decision support system. If the assessed data happen to be on target, the CPSMS will encourage the patient to follow the existing treatment plan, and continue to monitor and assess the patient's condition. The CPSMS system will iterate the assessment periodically to ensure the treat-to-target objective. The CPSMS can suggest that health personnel looks at the data and make changes to the treatment objectives or plan. It can make adjustments of the intensity of exercises, diet, and other non-pharmacological measures within the treatment plan. These changes can also be suggested before the target is missed, e.g., when a trend is observable. The inference engine can be applied to trigger different levels of alert according to each patient's disease condition. On the other hand, it can also notify health personnel to undertake medical assessment and change treatment plans or target objectives through face-to-face consultation such as making an appointment with professionals, or reducing the intake of a medicine. The computational models of the pathway will be used to support the automatic implementation of the recommended changes to treatment plan or targets.

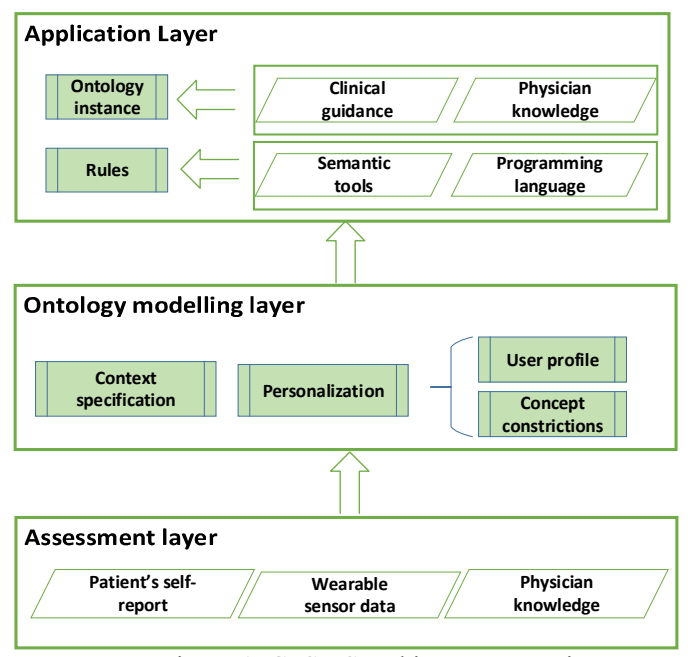

Figure 1. CPSMS architecture overview

\section{MODELS AND METHODS FOR CPSMS DECISION SUPPORT}

\section{A. Knowledge Acquisition and Conceptualization}

Knowledge acquisition is the process of extracting, structuring and organizing knowledge from human expert [20]. From a practical 
perspective, we use the domain knowledge of SpA from the following sources:

Firstly, the SpA assessment methods from the medical perspective, i.e. medical indices based testing, physiological vital measurements and patient self-reporting. For each method the measurements and metrics will also be captured. Medical index and physiological vital measurements are tested by sensor-based body movement detection which is to assess patient's disease conditions through non-clinical methods. Such methods provide measurable parameters that contain ASDAS [21] and BATH index in SpA selfassessment which includes measurement of Bath AS Metrology Index (BASMI) [22], Ankylosing Spondylitis Disease Activity Score (ASDAS) [23], Bath AS Functional Index (BASFI) [24], and Bath AS Disease Activity Index (BASDAI) [25]. Other clinical tests are 6 minute walk test, stair test, 30 second sit-to-stand, handgrip test, the modified fingertip-to-floor test. The physiological vital parameters can also be detected from biomedical sensors, i.e. heart rate, skin conductance, blood pressure, etc.

Secondly, the $\mathrm{SpA}$ patient rehabilitation pathway and assessment methods from the rehabilitation and monitoring perspective [26], i.e., the pathway of SpA patients from initial diagnosis to rehabilitation based on diet, medication and physical exercises, treat-to-target practice and rehabilitation effectiveness assessment methods and criteria.

Thirdly, the functions and underlying data and knowledge structures from the decision support perspective such as the formal definitions of various medical, physiological and behavioural measurements and metrics, identification and specification of core entities and concepts underpinning treat-to-target system. Such system includes the structure and computational presentation of a treatment plan, a treatment target, the pathway as well as the criteria and mechanism for assessing treat-to-target effectiveness.

This information is then classified and formalized using the commonly agreed vocabularies and terms, and described by a number of concepts, objects and the relationships among them in the ontology conceptualization. Classes or concepts, properties and property constraints are constructed manually in our system.

For the patient-specific conceptual model, the patient profile shown in Figure 2 uses, four main concepts which are "Assessment", "Target", "Treatment" and "Alert". These classes are connected via property constraints with the patient.

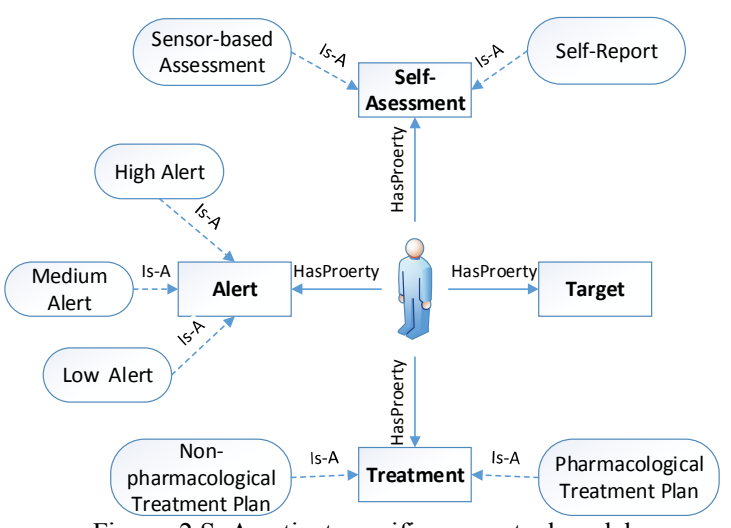

Figure. 2 SpA patient-specific conceptual model

An ontology conceptualization can be defined as an intentional semantic structure that encodes implicit knowledge constraining the structure of all entities within a domain. Such conceptualization should be expressed in a formal machine-readable format [27]. According to this idea, each class has its subclasses through ontological hierarchical relationship. For example, the class "Treatment" has two subclasses: "Non-pharmacological Treatment Plan" and "Pharmacological Treatment Plan", whilst these two subclasses also have their subclasses. In the class "Non-pharmacological Treatment Plan", there are subclasses "Education", "Exercises", "Join_group", "Massage" and "Weight reduction". The relationships between concepts are represented as properties. For instance, between the classes "patient" and "Assessment" a property named "hasAsssessment" exists, between "patient" and "Target" a property "hasTarget" exits, etc.

TABLE I. CPSMS CONCEPT, PROPERTIES AND INSTANCESS

\begin{tabular}{|l|l|l|}
\hline Concept & \multicolumn{1}{|c|}{ Properties } & Instances \\
\hline Patient & $\begin{array}{l}\text { hasAlert } \\
\text { hasAssessment } \\
\text { hasTarget } \\
\text { hasTreatment }\end{array}$ & $\begin{array}{l}\text { Mohn } \\
\text { Monica }\end{array}$ \\
\hline Assessment & $\begin{array}{l}\text { hasQuestionnaires } \\
\text { hasDevice }\end{array}$ & $\begin{array}{l}\text { BASDAI } \\
\text { BASMI } \\
\text { BASFI }\end{array}$ \\
\hline Target & $\begin{array}{l}\text { hasNon- } \\
\text { pharmacological_plan } \\
\text { hasPharmacological_ } \\
\text { plan }\end{array}$ & $\begin{array}{l}\text { Education } \\
\text { Exercise } \\
\text { Drugs }\end{array}$ \\
\hline hastert & $\begin{array}{l}\text { hasHent } \\
\text { hasMediumAlert } \\
\text { hasLowAlert. }\end{array}$ & $\begin{array}{l}\text { Change target } \\
\text { Change plan }\end{array}$ \\
\hline
\end{tabular}




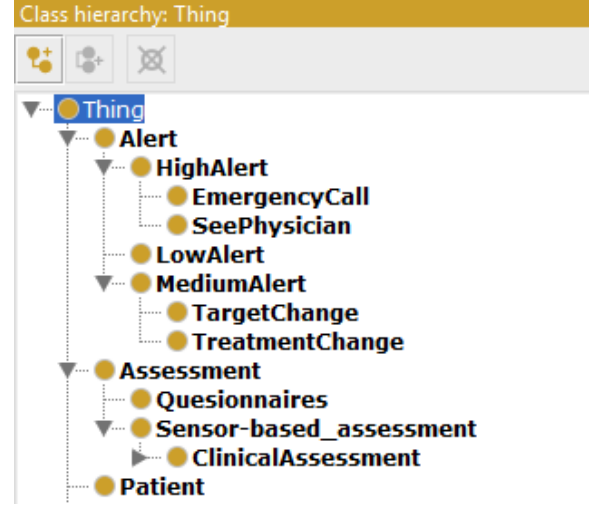

Figure.3 part of CPSMS clinical ontology model in protégé

Figure 3 presents the CPSMS ontological model constructed in the protégé 5.0 and Table 1 displays these key concepts, definitions, their properties and instances identified in the system. For instance, for the class "Alert" three subclasses are defined, where "Low Alert" is activated when the assessment outcome is a few changes. "Medium Alert" would be triggered when the disease condition becomes worse following treatment plan. While all the assessment parameters become deteriorating, the "High Alert" will be activated, meaning the patient needs to see physicians.

\section{B. CPSMS decision making mechanism}

A Semantic Web Rule Language (SWRL) is a rule language defined by the semantic web consortium based on the combination of the OWL DL and OWL Lite sublanguages [28]. SWRL rules are defined in the form of an implication between an antecedent and consequent. The conditions are specified in the antecedent, and the results of the reasoning are declared in the consequent. Rules in the CPSMS are based on SpA treat-to-target principle. The defined rules determine if the alert would be activated and which level of alert should be triggered. For example, The "Medium Plan" can be divided into "TargetChange" and "TreatmentChange". Rule 1 expresses that if a patient takes both non-pharmacological treatment and pharmacological treatment, and assessment result is not on target, then the non-pharmacological treatment would be changed. Rule 2 denotes that if non-pharmacological treatment has been changed a few times, and self-assessment result is still not on target, then the target would be changed, meaning this target may be too high for the patient and change is needed. Rule 3 shows the high alert conditions that each index has an increase of more than its given threshold set to 1.3 respectively, the high alert will be triggered.

Rule1: Patient(?p), hasSpA(?p, ?spa), hasNonpharmacological_plan(?p, ?npp), hasPharmacological_plan(?p, ?pp), hasAssessment(?p, ?as), hasTarget(?p, ?t), lessThan(?as, ?t) $\quad \rightarrow \quad$ hasNonpharmacological_planChange(?p, ?nnpc)

Rule2: Patient(?p), hasSpA(?p, ?spa), hasNonpharmacological_plan(?p, ?npp), hasTarget(?p, ?t),
hasAssessment(?p, ?as1), hasAssessment(?p, ?as2), lessThan(?as2, ?as1) -> hasTreatmentChange(?p, ?tc)

Rule3: Patient(?p), hasSpA(?p, ?spa), hasBASMIScore(?p, ?bm), hasBASFIScore(?p, ?bf), hasBASDAI (?p, ?ba), greaterThanOrEqual(?bm, 1.3$)$, greaterThanOrEqual(?bf, 1.3), greaterThanOrEqual(?ba, 1.3), -> hasHighAlert(?p, ?al)

\section{CPSMS implementation}

Figure 4 depicts the CPSMS execution process. A patient uses sensors to detect the physical movement inputs, and also fills out the questionnaires for each index including ASDAS, BASDAI, BASMI, BASFI produce a score out of 10. Then collected data will be processed by the mathematical assessment model and then stored in a database in the self-assessment module. The CPSMS ontological model including concepts and their relationships are able to be instantiated based on patient medical measurements, behavioural observations and profile in these two steps. The SWRL rules are applied in the decision support module to compare the outcome from selfassessment module to the specified target. If the assessment indicates that the result after treatment is not on target, then the treatment plan or target would be suggested to be changed according to patient's condition. If the outcomes from each clinical index and general assessment from selfassessment module show that the patient is in a severe condition, then the high alert will be triggered to notify physicians, whilst the system would suggest patient to go to a clinical centre.

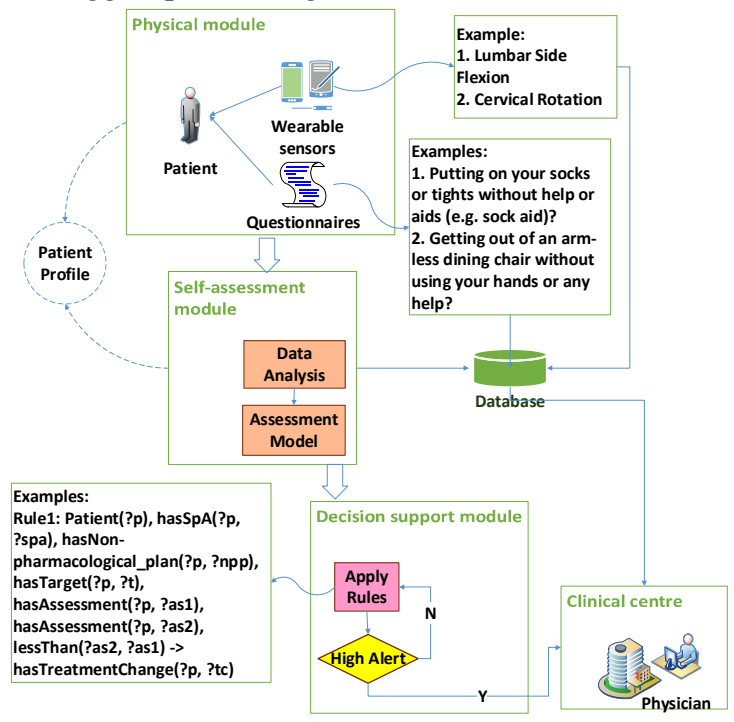

Figure 4. Process of the CPSMS execution

The system uses Pellet [29] as OWL reasoner embedded in the Java code to apply the rules. The Pellet plugin can produce DL queries in protégé using Manchester OWL syntax, and it supports the SWRL engine in protégé. Using the Java development tool, the Pellet plugin can not only 
play the same role as in the protégé, but are capable of processing the ontology through creating instances, data properties, object properties and data types. Furthermore, once a reasoner instance has been obtained, the reasoner can be queried for information about the constructed ontology through making use of SWRLQueryAPI. With the fully automatic decision support system for chronical patient controlling their disease at home, each outcome of measurement and the self-assessment from relational database will be sent to the clinical centre for the reference to physicians when the patient visiting them, contributing to the reduction in cost and process of re-measurement.

\section{USE SCENARIO}

To evaluate our concept, we use the following simplified scenario:

Mark is a SpA patient, male, 25 years old. His SpA disease was confirmed one year ago, and now it is under stable condition. He is used to see doctor every second week to control his disease. The most obvious symptom for him is back pain and morning stiffness. Usually doctor prescribes pharmacological treatment.

With the assistance of the home-based selfmanagement system, he is able to monitor his disease condition on his own. The CPSMS provides Mark with his condition information and both pharmacological and non-pharmacological treatment plans. Using the CPSMS, he can reduce the visits to the physician to once a month or when recommend by the CPSMS. When using the CPSMS, he first fills out the BASFI, BASDAI, and ASDAS questionnaires. Assume that the outcomes are 3, 4, and 2.1, 2.5 respectively, and the BASMI score is 5 measured by the sensors. The outcomes of each index suggest that Mark's condition is not severer than at the last measurement. However, his result is difference to the last measurement. Mark follows doctor's pharmacological and nonpharmacological treatment plans, but the disease condition is still not even getting better. Hence, the CPSMS automatically updates non-pharmacological plan in terms of his severe part: back pain and morning stiffness. Regular exercise including home exercises and physical therapies are recommended as shown in Rules 1 and 2 below. The concrete exercise rules are shown in rule 3-5. Rule 3 denotes that when Mark's ASDAS and BATH index become worse than the last assessment result, the system would recommend him to add 15 minutes extra training in pool. Rule 4 denotes that if Mark's other exercises taken outside pool are more than 30 minutes, then he is suggested to add 20 minutes pool training on the following week and stop exercise next day. Rule 5 is a case when each assessment index becomes better, Mark would be suggested to take more walking and less pool training.

Rule 1: Patient(?Mark), hasSpA(?Mark, ?spa), hasNon-pharmacological_plan(?Mark, ?npp), hasPharmacological_plan(?Mark, ?pp),
hasAssessment(?Mark, ?as), hasTarget(?Mark, ?t), lessThan(?as, ?t) $\quad \rightarrow$ hasNonpharmacological_planChange(?Mark,?nnpc)

Rule 2: Patient(?Mark), hasSpA(?Mark, ?spa), hasNon-pharmacological_planChange(?Mark,

?nnpc), hasBackPain(?mark, ?bp), hasMorningStiff(?Mark, ?ms) -> addRegularExercise(?Mark, ?re)

Rule 3: Patient(?Mark), hasSpA(?Mark, ?spa), hasASDAS(?Mark, ?as2), hasBASDAI(?Mark, ?ba2), hasBASFI(?Mark, ?bf2), hasBASMI(?Mark, ?bf2), greaterThan(?as2, ?as1), greaterThan (?ba2, ?ba1), greaterThan(?bf2, ?bf1) -> addExerciseTime(?Mark, ?poolTrainingTime), Equal(?poolTrainingTime, 15).

Rule 4: Patien(?Mark), hasSpA(?Mark, ?spa), hasExerciseTime(?Mark, ?poolTrainingTime), greaterThan(?poolTrainingTime, 30) -> addExerciseTime(?Mark, ?poolTrainingTime), hasTime(?Mark, ?2nd week), Equal(?poolTrainingTime, 20), reduceExerciseTime(?Mark, 0), hasTime(?Mark, ?2nd day).

Rule 5: Patient(?Mark), hasSpA(?Mark, ?spa), hasAssessment(?Mark, as2), hasAssessment(?Mark, ?as1), lessThan(?as2, ?as1) -> reduceExerciseTime(?Mark, ?poolTrainingTime), addExerciseTime(?Mark, ?walking).

Following the new treatment, Mark's condition is becoming a bit better on the second week, thus the CPSMS keeps these plans and encourages Mark continually to follow these until next assessment.

\section{CONCLUSION AND FUTURE WORK}

To improve the quality of life for patients with chronic diseases and reduce unnecessary resources consumption, we developed an ontology-based decision support system that facilitates patients with chronic disease to carry out personalized selfmanagement. In this method, the ontological models are constructed, and the patient profile instances are produced to apply formalized SWRL rules for decision making. A number of rules are created to model and represent the treat-to-target principles which are in turn used to assess the patients' symptoms to trigger different level alerts in residential environments. This method has been illustrated for SpA patients, but these can be extended to other diseases and use cases. In our further work, the method will be applied to a more realistic medical setting for SpA-patients, specifically addressing the problem that such patients face with physical training: too much training can affect these patients as much as too little training. Based on personalised rules for training and using a disease- and training diary, the patients will be given recommendations to handle their disease in self-management.

\section{ACKNOWLEDGMENT}

This work has been supported by the Mobile Musculoskeletal User Self-management (MOSKUS) project funded by Research Council of 
Norway in the VERDIKT programme, grant number 227251. We would like to thank Kåre Birger Hagen, Dag Soldal, Nina Østerås, Camilla Fongen, and Carl-Henrik R. Franke for sharing their medical expertise with us.

\section{REFERENCE}

[1] Busse, Reinhard. Tackling chronic disease in Europe: strategies, interventions and challenges. No. 20. WHO Regional Office Europe, 2010.

[2] Lisac, Melanie, et al. "Health Systems and Health Reform in Europe."Intereconomics 43.4 (2008): 184-218.

[3] Gangemi A , Steve G, Giacomelli F, "ONIONS: An Ontological Methodology for Taxonomic Knowledge Integration" In P. van der Vet (ed.) Proceedings of the Workshop on Ontological Engineering, ECAI96, 1996.

[4] McGuinness, Deborah L. "Ontological issues for knowledgeenhanced search."Proceedings of Formal Ontology in Information Systems. 1998.

[5] Antoniou, Grigoris, and Frank Van Harmelen. "Web ontology language: Owl."Handbook on ontologies. Springer Berlin Heidelberg, 2004. 67-92.

[6] Abidi, Samina R., et al. "Ontology-based modeling of clinical practice guidelines: a clinical decision support system for breast cancer follow-up interventions at primary care settings." (2007): 845.

[7] RiañO, David, et al. "An ontology-based personalization of health-care knowledge to support clinical decisions for chronically ill patients." Journal of biomedical informatics 45.3 (2012): 429-446.

[8] Paganelli, Federica, and Dino Giuli. "An ontology-based context model for home health monitoring and alerting in chronic patient care networks."Advanced Information Networking and Applications Workshops, 2007, AINAW'07. 21st International Conference on. Vol. 2. IEEE, 2007.

[9] Thomas, Karl W., Charles S. Dayton, and Michael W. Peterson. "Evaluation of internet-based clinical decision support systems." Journal of Medical Internet Research 1.2 (1999).

[10] Martínez-García, Alicia, et al. "Sharing clinical decisions for multimorbidity case management using social network and open-source tools." Journal of biomedical informatics 46.6 (2013): 977-984

[11] EGADSS: http://www.egadss.org

[12] Gangemi A , Steve G, Giacomelli F, "ONIONS: An Ontological Methodology for Taxonomic Knowledge Integration" In P. van der Vet (ed.) Proceedings of the Workshop on Ontological Engineering, ECAI96, 1996.

[13] Steve G, Gangemi A, "ONIONS Methodology and Ontological Commitment of Medical Ontology ON8.5", Proceedings of Knowledge Acquisition Workshop, Banff, participants edition, 1996.

[14] Steve G, Gangemi A, Pisanelli DM, "Integrating Medical Terminologies with ONIONS Methodology", in Kangassalo H, Charrel JP (eds.) Information Modelling and Knowledge Bases VIII, Amsterdam, IOS Press 1997.

[15] The Open Biological and Biomedical Ontologies: < http://www.obofoundry.org/>

[16] OGMS: <https://code.google.com/p/ogms>

[17] Smith, Barry, et al. "The OBO Foundry: coordinated evolution of ontologies to support biomedical data integration." Nature biotechnology 25.11 (2007): 12511255.

[18] Garde, Sebastian, et al. "Expressing clinical data sets with openEHR archetypes: a solid basis for ubiquitous computing." International journal of medical informatics 76 (2007): S334-S341.

[19] Smolen, Josef S., et al. "Treating spondyloarthritis, including ankylosing spondylitis and psoriatic arthritis, to target: recommendations of an international task force." Annals of the rheumatic diseases 73.1 (2014): 6-16.

[20] Liou, Yihwa Irene. "Knowledge acquisition: issues, techniques, and methodology." Proceedings of the 1990 ACM SIGBDP conference on Trends and directions in expert systems. ACM, 1990.

[21] van der Heijde, Désirée, et al. "ASDAS, a highly discriminatory ASAS-endorsed disease activity score in patients with ankylosing spondylitis." Annals of the rheumatic diseases 68.12 (2009): 1811-1818.

[22] Jenkinson, Tim R., et al. "Defining spinal mobility in ankylosing spondylitis (AS). The Bath AS Metrology Index." The Journal of Rheumatology 21.9 (1994): 16941698.

[23] Garrett, Sarah, et al. "A new approach to defining disease status in ankylosing spondylitis: the Bath Ankylosing Spondylitis Disease Activity Index." The Journal of Rheumatology 21.12 (1994): 2286-2291.

[24] Calin, Andrei, et al. "A new approach to defining functional ability in ankylosing spondylitis: the development of the Bath Ankylosing Spondylitis Functional Index." The Journal of rheumatology 21.12 (1994): 2281-2285.

[25] Garrett, Sarah, et al. "A new approach to defining disease status in ankylosing spondylitis: the Bath Ankylosing Spondylitis Disease Activity Index." The Journal of Rheumatology 21.12 (1994): 2286-2291.

[26] Hreggvidsdottir, Hulda S., Troy Noordenbos, and Dominique L. Baeten. "Inflammatory pathways in spondyloarthritis." Molecular immunology 57.1 (2014): 28-37.

[27] Giaretta, Pierdaniele, and N. Guarino. "Ontologies and knowledge bases towards a terminological clarification." Towards Very Large Knowledge Bases: Knowledge Building \& Knowledge Sharing 1995 (1995): 25-32.

[28] Horrocks, Ian, et al. "SWRL: A semantic web rule language combining OWL and RuleML." W3C Member submission 21 (2004): 79

[29] Sirin, Evren, et al. "Pellet: A practical owl-dl reasoner." Web Semantics: science, services and agents on the World Wide Web 5.2 (2007): 51-53. 